\title{
Application of fractal dimension analysis and photodynamic diagnosis in the case of differentiation between lichen planus and leukoplakia: A preliminary study
}

\author{
Kamil Jurczyszyn ${ }^{1, A, C, D}$, Klaudia Kazubowska ${ }^{1, B}$, Paweł Kubasiewicz-Ross ${ }^{1, D}$, Piotr Ziółkowski2,E, Marzena Dominiakk ${ }^{1, F}$ \\ ${ }^{1}$ Department of Oral Surgery, Faculty of Dentistry, Wroclaw Medical University, Poland \\ 2 Department of Pathology, Faculty of Medicine, Wroclaw Medical University, Poland \\ A - research concept and design; $B$ - collection and/or assembly of data; $C$ - data analysis and interpretation; \\ $\mathrm{D}$ - writing the article; $\mathrm{E}$ - critical revision of the article; $\mathrm{F}$ - final approval of the article
}

Address for correspondence

Kamil Jurczyszyn

E-mail: kjurczysz@interia.pl

Funding sources

Grant for young scientists PBMN152

Conflict of interest

None declared

Received on April 19, 2017

Reviewed on October 5, 2017

Accepted on November 27, 2017

\begin{abstract}
Background. Photodynamic therapy (PDT) is a noninvasive method for the treatment of premalignant lesions, such as leukoplakia and lichen planus (LP). These lesions are very irregular. In the case of such irregular lesions, fractal dimension analysis (FDA) is very helpful. Photodynamic diagnosis (PDD) enables the visualization of irregular lesion shapes more precisely than a classical white-light examination.
\end{abstract}

Objectives. In our study, we tried to distinguish oral leukoplakia and LP, using FDA in a classical examination with white light and PDD. Lesions treated using PDT were histopathologically verified.

Material and methods. We enrolled 35 patients in our study. Fractalyse software v. 2.4 (University of Franche-Comté, Besançon, France) was used to count fractal dimensions (FDs). Photodynamic therapy and PDD were mediated with 20\% delta-aminolevulinic acid (5-ALA).

Results. Fractal dimensions of leukoplakia foci of the tongue in a white-light examination were significantly lower than in PDD. In the case of LP, a significant difference of FDs was observed between lesions in the cheek and in the alveolar ridge region. Differences in FDs were observed between leukoplakia foci of the alveolar ridge, tongue and palate. A complete response of leukoplakia foci to PDT was observed in 10 out of 34 lesions, partial remission occurred in 20 lesions and a total lack of response was noted in 4 lesions. Generally, LP was completely treated in 7 out of 14 cases, a partial response was observed in 5 lesions and a failure of PDT treatment was noted in 2 cases.

Conclusions. Fractal dimension analysis may be a useful method in the comparison of complicated shapes of such lesions as LP or leukoplakia, but our study did not confirm that this method may be used to distinguish LP and leukoplakia without a histopathological examination. Photodynamic therapy is an effective treatment method in the case of $L P$ and leukoplakia of the oral cavity.

Key words: photodynamic therapy, lichen planus, leukoplakia, fractal dimension analysis, photodynamic diagnosis

DOI

10.17219/acem/80831

Copyright

Copyright by Author(s)

This is an article distributed under the terms of the

Creative Commons Attribution Non-Commercial License

(http://creativecommons.org/licenses/by-nc-nd/4.0/) 


\section{Introduction}

Photodynamic therapy (PDT) is a noninvasive treatment method for premalignant and malignant lesions. This therapy is commonly used in dermatology for the treatment of solar keratosis, actinic keratosis, Bowen's disease, and basal cell carcinoma. ${ }^{1-4}$ Photodynamic therapy is composed of 2 main agents: light and a photosensitizer (PS). It has to be emphasized that the doses of light and PS are too weak to manage the clinical effect separately; only the combination of these 2 agents is responsible for the effect of the treatment. One of the most important features of PS is an affinity for cells with higher metabolism. After penetrating the cell membrane, PS accumulates in the target cells. Delta-aminolevulinic acid (5-ALA) is one of the most commonly used precursors of PS in PDT. After penetrating the cells, 5-ALA passes into the biochemical pathways of heme. Protoporphyrin IX (PPIX) is the effect of these reactions; it is also a proper photosensitizer. Protoporphyrin IX has few peaks in the spectrum of absorption. The first and the highest peak of absorption - at the $405 \mathrm{~nm}$ wavelength - is called Sorret's band. It is used during the procedure of photodynamic diagnosis (PDD). The irradiation of PPIX in Sorret's band leads to red fluorescence and phosphorescence inside the cells. This phenomenon is used during PDD. Unmetabolized 5-ALA is unable to fluoresce after $405 \mathrm{~nm}$ excitation. It prevents false-positive trials during PDD. Higher wavelengths are used in the case of PDT, because skin and mucous membrane penetration by light increases along with the longer waves of light in the visible and near infrared (vis-NIR) electromagnetic spectrum. Because of this function, red light $(635-650 \mathrm{~nm})$ is used during PDT.

Photodynamic diagnosis very often enables the visualization of irregular lesion shapes more precisely than a classical white-light examination. ${ }^{5,6}$ In the case of hyperkeratotic lesions, during PDD, the fluorescence of healthy background tissue is higher than the fluorescence of pathological lesions due to the thicker layer of the epithelium; thus, in these cases a negative image of the examined lesion is observed.

Leukoplakia and lichen planus (LP) are mucous membrane lesions which are very difficult to treat when they are large or multifocal; these 2 lesions are also precancerous stages. The classic World Health Organization (WHO) definition of leukoplakia from 1978 characterizes it as "a white patch or plaque that cannot be characterized clinically or pathologically as any other disease". 7 The etiology of leukoplakia is multifactorial. The most important factors are cigarette smoking, alcohol consumption, poor oral hygiene, sharp edges of the teeth, defective fillings, electrogalvanic currents (due to various metals in the oral cavity, i.e., amalgam, gold or nickel), food irritation, or the oral mucosa.

Since 2002, it has been recommended to make a distinction between a provisional clinical diagnosis of oral leukoplakia and a definitive one. A provisional clinical diagnosis is made when a lesion at the initial clinical examination cannot be clearly diagnosed as either leukoplakia or any other disease. $^{8}$

The etiology of LP is still not fully known. According to the most common theories, it is a chronic, probably autoimmune, mucocutaneous, psychosocial disease that usually presents in middle-aged females and primarily affects the oral mucosa, skin, genital mucosa, scalp, and nails. Oral LP can clinically present in various forms, including reticular, papular, plaque-like, atrophic, erosive, and bullous types. ${ }^{9}$

The characteristic feature of both abovementioned lesions is a very irregular shape, therefore it is very hard to measure the area of these lesions. In the case of such irregular lesions, fractal dimension analysis (FDA) is very helpful. Fractal dimension analysis is a very promising method which is widely used to describe complicated shapes when the classical methods fail.

The term "fractal" refers to a shape which is described by potentially simple mathematic formulas. If these formulas are iterated into infinity, they may create shapes which we are able to magnify indefinitely and each time we can see infinite numbers of details of the shape - it has the feature of self-similarity. In classical Euclidean geometry, dimension is an integer - it is a number of coordinates which we need to describe the point inside the shape. For example, a point has no dimension, so it equals 0 ; to describe a straight line we need only 1 dimension (length); the main features of a rectangle are its length and width; a 3-dimensional shape needs to have width, length and height. Classic examples of fractals are Cantor set, Koch snowflake and Sierpinski triangle (Fig. 1).

The fractal dimension (FD) of Cantor set equals approx.

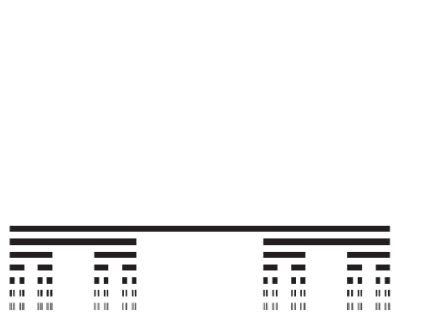

Cantor set

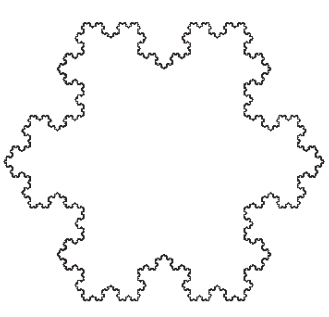

Koch snowflake

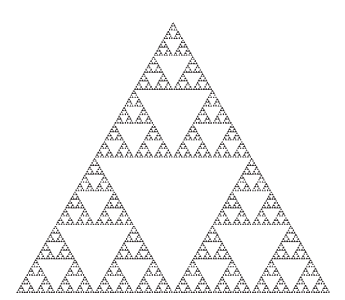

Sierpinski triangle
Fig. 1. Examples of fractals 
0.631. This means that this shape is something between a point and a line. Koch snowflake, with a FD $\approx 1.262$, is a shape which is closer to a line than to a flat figure, in contrast to Sierpinski triangle, with a FD $\approx 1.585$, which is nearly halfway between a line and a flat figure.

Some natural shapes may be considered fractals, e.g., coast lines, trees, clouds, and mountains. Examples of fractals in living organisms include nerves and branches of blood vessels, the structure of brain neurons and the structure of bone. These shapes are too complicated to measure or compare between each other using traditional methods based on Euclidean geometry. In such cases, FDA is non-substitutable.

It is important to mention that FDA offers the ability to compare complicated shapes. The value of FD describes only the distribution of points (on a surface or in space) which create these shapes, as opposed to traditional ways of physically describing the dimension of a shape.

Fractal dimension analysis can be very useful in medicine; examples of FDA usage in medicine are mammographic image analysis, or estimation of tumor neoangiogenesis or of the pattern of coronary vessels. ${ }^{10-12}$ Fractal dimension analysis of jaw bone cone beam computed tomography $(\mathrm{CBCT})$ images is useful in the diagnosis of osteoporosis. ${ }^{13}$

In our study, we tried to distinguish oral leukoplakia and LP using FDA with a classical white-light examination and PDD. Lesions treated using PDF were histopathologically verified.

\section{Material and methods}

\section{Patients}

We enrolled patients (20 females and 15 males) in our study. The mean age of the study group was 58 years (range: 32-81 years). The total number of patients suffering from leukoplakia was 26, while 9 patients suffered from LP. Each lesion was histopathologically examined after taking the specimen from pathologically changed oral mucosa under local anesthesia (a classical examination with hematoxylin and eosin (H\&E) staining). Leukoplakia foci occurred at the same rate in females and males. In the case of LP, females suffered from lesions more frequently than males (77.8\% vs $22.2 \%$, respectively). All procedures were conducted after obtaining the approval of the Ethics Committee of Wroclaw Medical University, Poland (approval No. KB-367/2014).

Leukoplakia foci were estimated using van der Waal classification. This classification is based on 3 parameters: L, C and P. The L parameter describes the size of the lesion: 1 - focus $\leq 2 \mathrm{~cm} ; 2$ - a lesion size $2-4 \mathrm{~cm}$; and 3 - a lesion size $>4 \mathrm{~cm}$. $\mathrm{L}_{\mathrm{X}}$ refers to an unspecified size. $\mathrm{C}$ is the clinical appearance of the lesion: 1 - homogenic; 2 - nonhomogenic. $\mathrm{P}$ describes the occurrence of dysplasia $\left(\mathrm{P}_{1}\right)$ in a histopathological examination or a non-dysplastic lesion $\left(\mathrm{P}_{0}\right) . \mathrm{P}_{\mathrm{X}}$ is the absence or presence of epithelial dysplasia not specified in the pathology report. According to these properties, van der Waal distinguishes 4 stages of leukoplakia: stage I $\left(\mathrm{L}_{1} \mathrm{P}_{0}\right)$; stage II $\left(\mathrm{L}_{2} \mathrm{P}_{0}\right)$; stage III $\left(\mathrm{L}_{3} \mathrm{P}_{0}\right.$ or $\left.\mathrm{L}_{1} \mathrm{~L}_{2} \mathrm{P}_{1}\right)$; or stage IV $\left(\mathrm{L}_{3} \mathrm{P}_{1}\right)$. In our study, we admitted only patients with homogenic leukoplakia without dysplasia $\left(\mathrm{L}_{1} \mathrm{P}_{0}, \mathrm{~L}_{2} \mathrm{P}_{0}\right.$, or $\left.\mathrm{L}_{3} \mathrm{P}_{0}\right)$. Other patients were treated by surgery. In the case of erosive LP, the patients were excluded from the study. All LP lesions were classified as reticular.

\section{Photodynamic therapy and photodynamic diagnosis procedure}

A solution of 20\% 5-ALA (Sigma-Aldrich, St. Louis, USA) was dissolved in an eucerin base directly before each procedure. Delta-aminolevulinic acid was applied to lesions and covered by an occlusive dressing (gelatinous sponge flakes). After $2 \mathrm{~h}$, PDD was performed. A Viofor-PDT lamp (Med \& Life, Komorów, Poland) was used as the source of light. To excite the photoensitizer, we used a 405-nanometer wavelength with $250 \mathrm{~mW}$ of power. Photos were taken using the same parameters: Canon EOS 500D (Canon Inc., Tokyo, Japan), a 13-millimeter intermediate ring, a 50-millimeter lens, orange and UVcut filters, an ISO of 1600, f-stop of $1 / 9$, and exposure time of $1 / 50 \mathrm{~s}$. Photo resolution was $4752 \times 3168$ pixels. After the PDD procedure, the 5-ALA ointment was applied again with an occlusive dressing for $2 \mathrm{~h}$. Next, PDT was performed using red light $(635 \mathrm{~nm}$, a Viofor PDT lamp) in a total dose of $120 \mathrm{~J}$ per lesion. All PDT procedures were repeated every 3 weeks for each patient. Patients were observed 3, 6 and 12 months after their last photodynamic procedure.

\section{Image preparation}

All graphical operations were performed in GIMP v. 2.8.0 (GNU Image Manipulation Program; www.gimp.org). In the center of the lesion, a square with 300 pixels per side was selected. Prepared image selection was cropped from the original photo. A high pass filter was applied and the Levels tools were used to equalize the histogram of the image. Next, the images were converted into a grayscale and then converted into bitmap images (with a 50\% threshold). The file was saved in TIFF format without any compression algorithms. All graphical operations for white-light photos are shown in Fig. 2. The PDD photos were prepared in the same way, but after the last bitmap transformation, color inversion was applied (Fig. 3). During PPD, hyperkeratotic lesions are darker than healthy mucosa, so color inversion was necessary to obtain pictures analogous to the white-light ones. These prepared files were the basis for FDA. 


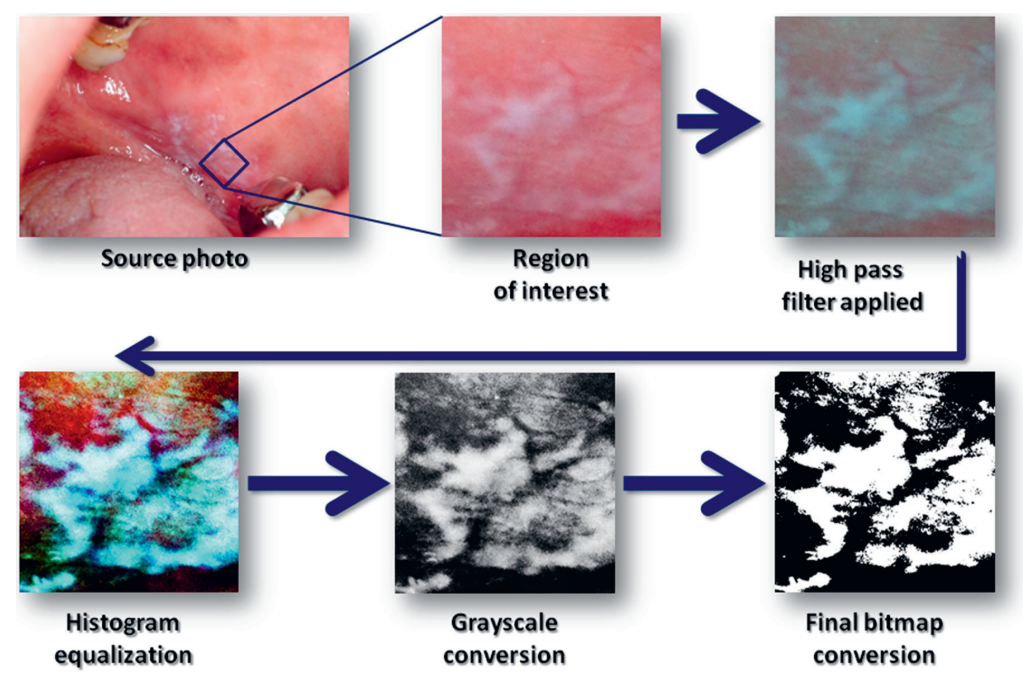

Fig. 2. Preparation of images during a white-light examination

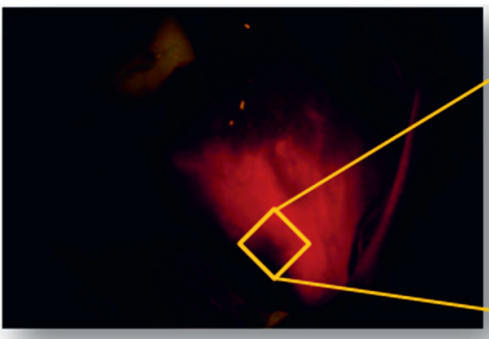

Source photo

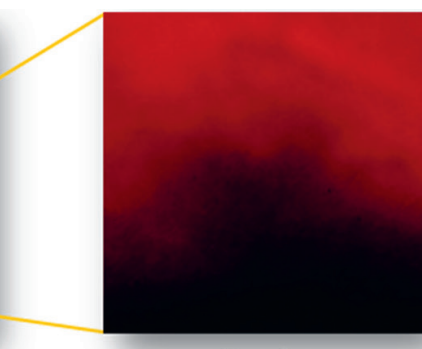

Region of interest

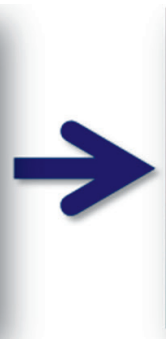

High pass filter applied

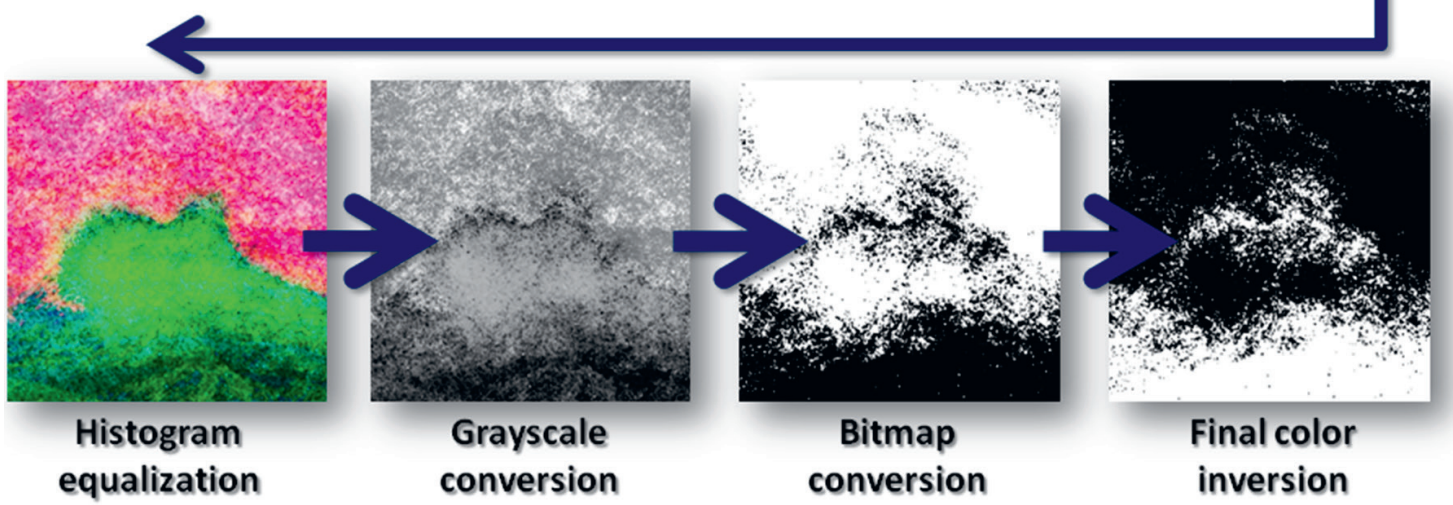

\section{Fractal dimension analysis}

We used the computer program Fractalyse v. 2.4 (University of Franche-Comté, Besançon, France). Fractalyse enables the user to measure FDs using the box-counting method. The fractal dimension $\left(D_{s}\right)$ is counted using the formula below ${ }^{14}$ :

$$
D_{S}=\lim _{\varepsilon \rightarrow 0} \frac{\log N(\varepsilon)}{\log \left(\frac{1}{\varepsilon}\right)}
$$

where $D_{s}$ - the fractal dimension; $\varepsilon$ - the length of the box which creates a mesh covering the surface with the examined pattern; $N(\varepsilon)$ - the minimal number of boxes required to cover the examined pattern.

\section{Statistical analysis}

GraphPad Prism v. 6.01 (GraphPad Software, La Jolla, USA) was used for the statistical analysis. The Shapiro-Wilk test was applied to check for normality. Due to the lack of normal distribution in the examined samples, we used a non-parametric test. The Mann-Whitney test was applied to compare 2 values of FD. In the cases of more than 2 FD values, we used the Kruskal-Wallis test with Dunn's multiple comparisons test. The significance level was set at 0.05 .

\section{Results}

The most frequent location of lesions was the mucous membrane of the cheeks: for leukoplakia, 15 lesions were 
located there and for LP there were 6 lesions. Another 5 lesions of LP occurred in the alveolar ridge. The floor of the oral cavity was affected by LP in 2 cases. Lichen planus very rarely occurred on the tongue - only 1 lesion - and was not observed in the region of the lips or the hard palate. Leukoplakia lesions occurred in the tongue area in 7 cases and in 5 cases, in the alveolar ridge. Leukoplakia was very rarely observed in the region of the hard palate (3 lesions), the floor of the oral cavity (2 lesions) or the lips (2 lesions).

Stage L2 was most commonly observed (41.2\%), L1 lesions occurred in $35.3 \%$ of cases and L3 occurred least often (23.5\%).
The differences between the FDs of leukoplakia and LP in a white-light examination and the PDD procedure are shown in Tables 1 and 2. It is important to note that in the case of leukoplakia, the FD of lesions in the tongue in a white-light examination was significantly lower than during PDD. This means that lesions in PDD seem to be larger in both dimensions. No other FDs show statistically significant differences.

There were no significant differences observed between the FDs of leukoplakia and LP in the case of a white-light, classical examination or PDD. These results are shown in Tables 3 and 4.

Table 1. Fractal dimension values of leukoplakia in a white-light examination and the PDD procedure (Mann-Whitney test)

\begin{tabular}{|c|c|c|c|c|c|c|c|}
\hline \multirow{3}{*}{ Site } & \multicolumn{6}{|c|}{ Leukoplakia } & \multirow{3}{*}{$\mathrm{p}$-value } \\
\hline & \multicolumn{3}{|c|}{ white light } & \multicolumn{3}{|c|}{ PDD } & \\
\hline & median & mean & SD & median & mean & SD & \\
\hline Cheek & 1.780 & 1.793 & 0.029 & 1.808 & 1.795 & 0.049 & 0.271 \\
\hline Alveolar ridge & 1.805 & 1.811 & 0.027 & 1.857 & 1.854 & 0.011 & 0.057 \\
\hline Palate & 1.746 & 1.718 & 0.049 & 1.634 & 1.640 & 0.144 & 0.600 \\
\hline Tongue & 1.744 & 1.744 & 0.009 & 1.773 & 1.769 & 0.014 & 0.026 \\
\hline All locations & 1.773 & 1.774 & 0.042 & 1.782 & 1.780 & 0.084 & 0.162 \\
\hline
\end{tabular}

PDD - photodynamic diagnosis; SD - standard deviation.

Table 2. Fractal dimension values of lichen planus in a white-light examination and the PDD procedure (Mann-Whitney test)

\begin{tabular}{|c|c|c|c|c|c|c|c|}
\hline \multirow{3}{*}{ Site } & \multicolumn{6}{|c|}{ Lichen planus } & \multirow{3}{*}{$p$-value } \\
\hline & \multicolumn{3}{|c|}{ white light } & \multicolumn{3}{|c|}{ PDD } & \\
\hline & median & mean & SD & median & mean & SD & \\
\hline Cheek & 1.760 & 1.766 & 0.029 & 1.783 & 1.778 & 0.008 & 0.195 \\
\hline Alveolar ridge & 1.823 & 1.819 & 0.023 & 1.806 & 1.803 & 0.005 & 0.314 \\
\hline All locations & 1.780 & 1.787 & 0.038 & 1.787 & 1.787 & 0.016 & 0.914 \\
\hline
\end{tabular}

PDD - photodynamic diagnosis; SD - standard deviation.

Table 3. Values of fractal dimension of leukoplakia and lichen planus during a white-light examination (Mann-Whitney test)

\begin{tabular}{|c|c|c|c|c|c|c|c|}
\hline \multirow{3}{*}{ Site } & \multicolumn{6}{|c|}{ White light } & \multirow{3}{*}{$p$-value } \\
\hline & \multicolumn{3}{|c|}{ leukoplakia } & \multicolumn{3}{|c|}{ lichen planus } & \\
\hline & median & mean & $\mathrm{SD}$ & median & mean & SD & \\
\hline Cheek & 1.780 & 1.793 & 0.029 & 1.760 & 1.766 & 0.029 & 0.068 \\
\hline Alveolar ridge & 1.805 & 1.811 & 0.027 & 1.823 & 1.819 & 0.023 & 0.629 \\
\hline All locations & 1.773 & 1.774 & 0.042 & 1.780 & 1.787 & 0.038 & 0.452 \\
\hline
\end{tabular}

SD - standard deviation.

Table 4. Values of fractal dimension of leukoplakia and lichen planus during PDD (Mann-Whitney test)

\begin{tabular}{|c|c|c|c|c|c|c|c|}
\hline \multirow{3}{*}{ Site } & \multicolumn{6}{|c|}{ PDD } & \multirow{3}{*}{$\mathrm{p}$-value } \\
\hline & \multicolumn{3}{|c|}{ leukoplakia } & \multicolumn{3}{|c|}{ lichen planus } & \\
\hline & median & mean & SD & median & mean & $\mathrm{SD}$ & \\
\hline Cheek & 1.808 & 1.795 & 0.049 & 1.783 & 1.778 & 0.008 & 0.196 \\
\hline Alveolar ridge & 1.857 & 1.854 & 0.011 & 1.806 & 1.803 & 0.005 & 0.057 \\
\hline All locations & 1.782 & 1.780 & 0.084 & 1.787 & 1.787 & 0.016 & 0.899 \\
\hline
\end{tabular}

PDD - photodynamic diagnosis; SD - standard deviation. 
Table 5. Differences in fractal dimension of lichen planus between the cheek and the alveolar ridge during a white-light examination and PDD (Mann-Whitney test)

\begin{tabular}{|c|c|c|c|c|c|c|c|}
\hline \multirow{3}{*}{ Diagnostic method } & \multicolumn{6}{|c|}{ Lichen planus } & \multirow{3}{*}{$\mathrm{p}$-value } \\
\hline & \multicolumn{3}{|c|}{ cheek } & \multicolumn{3}{|c|}{ alveolar ridge } & \\
\hline & median & mean & SD & median & mean & SD & \\
\hline White light & 1.760 & 1.766 & 0.029 & 1.823 & 1.819 & 0.023 & 0.019 \\
\hline PDD & 1.783 & 1.778 & 0.008 & 1.806 & 1.803 & 0.005 & 0.036 \\
\hline
\end{tabular}

PDD - photodynamic diagnosis; SD - standard deviation.

Table 6. Statistical differences between foci of leukoplakia in various locations according to the examination method (Dunn's multiple comparisons test)

\begin{tabular}{|c|c|c|c|c|}
\hline \multirow{3}{*}{ Dunn's multiple comparisons test } & \multicolumn{4}{|c|}{ Leukoplakia } \\
\hline & \multicolumn{2}{|c|}{ White light } & \multicolumn{2}{|c|}{ PDD } \\
\hline & mean rank diff. & significant: yes or no & mean rank diff. & significant: yes or no \\
\hline Cheek vs alveolar ridge & -2.95 & no & -7.80 & no \\
\hline Cheek vs palate & 10.88 & no & 7.87 & no \\
\hline Cheek vs tongue & 10.13 & yes & 5.87 & no \\
\hline Alveolar ridge vs palate & 13.83 & yes & 15.67 & yes \\
\hline Alveolar ridge vs tongue & 13.08 & yes & 13.67 & yes \\
\hline Palate vs tongue & -0.75 & no & -2.00 & no \\
\hline
\end{tabular}

In the case of LP, a significant difference in FD was observed between lesions in the cheek and the alveolar ridge region. The values of FD were greater in the region of the alveolar ridge in both white-light and PDD examination. These results are shown in Table 5.

Differences in the FD were observed between the leukoplakia foci of the alveolar ridge, the tongue and the palate. These differences occurred in both white-light and PDD. Different values of FD were observed between cheek and tongue lesions in a white-light examination, in contrast to PDD, where the values were similar. The results of the Kruskal-Wallis and Dunn's multiple comparisons tests are shown in Table 6.

In all leukoplakia foci, in the region of the palate and the floor of the mouth, we observed a complete response. In the region of the tongue, a complete response was observed in 4 lesions and a partial response in 3 lesions. Leukoplakia of the alveolar ridge was completely treated in the case of 2 lesions, a partial response was achieved in 2 lesions and a lack of therapeutic effect was observed in the case of 1 lesion. Lesions in the region of the cheeks were the most resistant to PDT. Only in the case of 2 lesions we observed a complete response, a partial response was observed in the case of 11 lesions and a lack of therapeutic results occurred in the case of 2 lesions. Overall, a complete response of leukoplakia foci to PDT observed in $29.4 \%$ cases, a partial remission occurred in $58.8 \%$ cases and a total lack of treatment was noted in $11.8 \%$ cases.

Complete treatment was achieved in the case of 1 LP lesion in the tongue area. In the alveolar ridge, 4 out of 5 lesions were completely treated and 1 was partially treated. In the region of the floor of the mouth, a complete response was noted in the case of 1 lesion and a partial response occurred in 1 lesion. The most resistant areas to PDT treatment were the cheeks, where complete response was seen in 1 lesion, a partial response was observed in 4 lesions and a lack of treatment effects was seen in 1 lesion. Generally, LP was completely treated in $50 \%$ of all cases (7 lesions), a partial response was observed in $35.7 \%$ of cases (5 lesions) and failed PDT treatment was noted in 14.3\% of cases (2 lesions). After PDT application, symptoms of LP, such as a burning pain, sensitivity to spicy foods and discomfort during speaking, disappeared in all of our patients, even if the lesion did not respond or only partially responded to the treatment.

\section{Discussion}

The diagnosis and treatment of leukoplakia and LP as premalignant lesions are important therapeutic problems. Photodynamic diagnosis allows the visualization of lesions in much more detail than a normal white-light examination. In many cases, the actual size and range of lesions are larger during a PDD session. This is particularly important in neoplasm lesions, where a margin of healthy tissue should be preserved. Due to the complicated shapes of leukoplakia and LP, FDA appears to be the most efficient method for estimating the size of these lesions. Fractal dimension analysis may be useful to check the microvascular pattern of LP in various locations of the oral cavity. Lucchese et al. showed that the FD of LP microvascular pattern is higher in buccal mucosa (1.167) and in the tongue mucosa (1.196) in comparison to healthy mucosa (1.123). ${ }^{15}$

No statistical differences were found in our study between the FDs of leukoplakia and LP. However, statistical 
differences within the groups were observed. The value of FD in a white-light examination and in PDD was lower in the cheek region than in the alveolar ridge. This means that lesions in the cheek region were smaller in 1 dimension (width or height) than in the alveolar ridge. Differences in the FD of leukoplakia were mainly observed in the tongue region during both white-light examination (1.744) and PDD examination (1.773). This suggests that tongue lesions during PDD are larger and that the shape is more complicated than during a classical examination. Fractal dimension analysis may be a useful method for comparing complicated shapes, such as those of LP or leukoplakia, though our study did not confirm the usefulness of this method for distinguishing LP and leukoplakia without a histopathological examination.

The clinical detection of leukoplakia facilitates autofluorescence, chemiluminescence or vital staining with toluidine blue, though these methods have relatively low specificity and are not recommended for distinguishing leukoplakia from other lesions. ${ }^{16}$ Another option is optical coherence tomography (OCT), which detects dysplasia by the fluctuation of light scattering due to random cellular changes in dysplastic tissues in comparison to normal mucosa. ${ }^{17}$ Another study proved that narrowband imaging (NBI) demonstrating an intraepithelial papillary capillary loop (IPCL) pattern destruction or a twisted elongation are indicative of histological changes in oral leukoplakia. ${ }^{18}$ Application of 5\% Lugol's solution aids in the featuring of suspicious lesions. Normal mucosa stains brown because of the high glycogen content, whereas leukoplakia appears pale compared to the surrounding normal tissue. ${ }^{19}$

Biopsy of the lesion and a histopathological examination still remains the standard diagnostic procedure for suspicious lesions. One of the possible features of leukoplakia is dysplasia, which manifests as architectural changes within the epithelial strata, combined with cellular atypia due to inappropriate differentiation.

The diagnostic process of LP is similar to the previously described diagnostic process of leukoplakia; it involves a provisional clinical diagnosis and histopathological confirmation. Lichen planus clinically presents mostly as one of 2 forms: reticular or erosive. ${ }^{20}$ The reticular form occurs more frequently and is usually asymptomatic. The erosive form is less common, but is more symptomatic. Symptoms may range from a slight discomfort to an intense pain that interferes with chewing. ${ }^{20,21}$ Our study revealed that after PDT application, these symptoms disappeared in all patients, even when the lesion did not respond or only partially responded to treatment.

Lichen planus and leukoplakia very frequently occur on a large area of the mucous membrane, which leads to complicated surgical treatment and requires reconstruction of the mucosa after complete excision. Surgical excision creates contracted scars, which may decrease patients' comfort. Photodynamic therapy as a noninvasive procedure is more frequently used for the treatment of leukoplakia and LP. ${ }^{22-27}$

Maloth et al. revealed that in their study, in the leukoplakia group, $17 \%$ of cases showed a complete response, $66 \%$ showed a partial response and $17 \%$ of the lesions did not respond to the treatment. In the LP group, $80 \%$ of the lesions showed a partial response and $20 \%$ did not respond. ${ }^{28}$ Those results are similar to our study in the case of leukoplakia, in contrast to the lower effectiveness of PDT in LP found in our study. Pietruska et al. used chlorin-e6mediated PDT, and their results were similar to 5-ALAmediated PDT. ${ }^{29}$ In the case of leukoplakia, the results were as follows: $27.3 \%$ of cases with a total response, $50 \%$ of cases with a partial response and $22.7 \%$ with no effect. ${ }^{29}$ A study by Semkin et al. demonstrated that laser ablation may be another treatment method; successful results were seen in $42 \%$ of cases, but a recurrence of lesions was observed in $58 \%$ of cases. ${ }^{30}$

Photodynamic therapy and cryotherapy appear to be comparative treatment methods that may both serve as alternatives for the traditional surgical treatment of oral leukoplakia. The advantages of PDT are its minimal invasiveness and localized character, which prevents damage of collagenous tissue structures. Photodynamic therapy is more convenient for patients, less painful and more esthetically pleasing. ${ }^{31}$

\section{Conclusions}

Fractal dimension analysis may be a useful method for the comparison of complicated shapes, such as those of LP or leukoplakia, but our study did not confirm that this method may be used to distinguish LP and leukoplakia without a histopathological examination.

Photodynamic therapy is a promising, noninvasive treatment method of leukoplakia and LP in the region of the oral cavity.

After PDT application, symptoms of LP, such as a burning pain, sensitivity to spicy foods and discomfort during speaking, disappeared in all of our patients, even when the lesion did not respond or only partially responded to treatment.

\section{References}

1. Canavan TN, de la Feld SF, Huang C, Sami N. Photodynamic therapy effective for the treatment of actinic keratosis and basal cell carcinoma in bullous pemphigoid patients. Photodiagnosis Photodyn Ther. 2017;18:257-259. doi: 10.1016/j.pdpdt.2017.03.019

2. Vignion-Dewalle AS, Baert G, Devos L, et al. Red light photodynamic therapy for actinic keratosis using $37 \mathrm{~J} / \mathrm{cm}^{2}$ : Fractionated irradiation with $12.3 \mathrm{~mW} / \mathrm{cm}^{2}$ after 30 minutes incubation time compared to standard continuous irradiation with $75 \mathrm{~mW} / \mathrm{cm}^{2}$ after 3 hours incubation time using a mathematical modeling. Lasers Surg Med. 2017;49(7):686-697. doi: 10.1002/Ism.22665

3. Megna M, Fabbrocini G, Marasca C, Monfrecola G. Photodynamic therapy and skin appendage disorders: A review. Skin Appendage Disord. 2017;2(3-4):166-176. doi: 10.1159/000453273 
4. Griffin LL, Lear JT. Photodynamic therapy and non-melanoma skin cancer. Cancers (Basel). 2016;8(10). pii: E98.

5. Hillemanns P, Wimberger P, Reif J, Stepp H, Klapdor R. Photodynamic diagnosis with 5-aminolevulinic acid for intraoperative detection of peritoneal metastases of ovarian cancer: A feasibility and dose finding study. Lasers Surg Med. 2017;49(2):169-176. doi: 10.1002/lsm. 22613

6. Kata SG, Zreik A, Ahmad S, Chłosta P, Aboumarzouk O. Concurrent bladder cancer in patients undergoing photodynamic diagnostic ureterorenoscopy: How many lesions do we miss under white light cystoscopy? Cent European J Urol. 2016;69(4):334-340.

7. World Health Organization. Collaborative Reference Centre for Oral Precancerous Lesions Application of the International Classification Of Diseases to Dentistry and Stomatology. Geneva, Switzerland: WHO; 1978.

8. Awan $\mathrm{KH}$, Morgan PR, Warnakulasuriya S. Utility of chemiluminescence (ViziLite ${ }^{\mathrm{TM}}$ ) in the detection of oral potentially malignant disorders and benign keratoses. J Oral Pathol Med. 2011;40(7):541-544.

9. Gupta A, Mohan RP, Gupta S, Malik SS, Goel S, Kamarthi N. Roles of serum uric acid, prolactin levels, and psychosocial factors in oral lichen planus. J Oral Sci. 2017;59(1):139-146. doi: 10.2334/josnusd. 16-0219

10. Zyout I, Togneri R. A new approach for the detection of architectural distortions using textural analysis of surrounding tissue. Conf Proc IEEE Eng Med Biol Soc. 2016;2016:3965-3968. doi: 10.1109/EMBC. 2016.7591595

11. Saidov $T$, Heneweer $C$, Kuenen $M$, et al. Fractal dimension of tumor microvasculature by DCE-US: Preliminary study in mice. Ultrasound Med Biol. 2016;42(12):2852-2863. doi: 10.1016/j.ultrasmedbio.2016.08.001

12. Yipintsoi T, Kroll K, Bassingthwaighte JB. Fractal regional myocardial blood flows pattern according to metabolism, not vascular anatomy. Am J Physiol Heart Circ Physiol. 2016;310(3):351-364. doi: 10.1152/ ajpheart.00632.2015

13. Güngör E, Yildirim D, Çevik R. Evaluation of osteoporosis in jaw bones using cone beam CT and dual-energy X-ray absorptiometry. J Oral Sci. 2016;58(2):185-194. doi: 10.2334/josnusd.15-0609

14. Grizzi F, Russo C, Colombo P, et al. Quantitative evaluation and modeling of two-dimensional neovascular network complexity: The surface fractal dimension. BMC Cancer. 2005:8:14.

15. Lucchese A, Gentile E, Capone G, De Vico G, Serpico R, Landini G. Fractal analysis of mucosal microvascular patterns in oral lichen planus: A preliminary study. Oral Surg Oral Med Oral Pathol Oral Radiol. 2015;120(5):609-615. doi: 10.1016/j.0ooo.2015.06.029

16. Awan K, Yang Y, Morgan P, Warnakulasuriya S. Utility of toluidine blue as a diagnostic adjunct in the detection of potentially malignant disorders of the oral cavity - a clinical and histological assessment. Oral Dis. 2012;18(8):728-733.
17. Lee CK, Chi TT, Wu CT, Tsai MT, Chiang CP, Yang CC. Diagnosis of oral precancer with optical coherence tomography. Biomed Opt Express. 2012;3(7):1632-1646

18. Yang SW, Lee YS, Chang LC, Hwang CC, Chen TA. Diagnostic significance of narrow-band imaging for detecting high-grade dysplasia, carcinoma in situ, and carcinoma in oral leukoplakia. Laryngoscope. 2012;122(12): 2754-2761.

19. Petruzzi M, Lucchese A, Baldoni E, Grassi FR, Serpico R. Use of Lugol's iodine in oral cancer diagnosis: An overview. Oral Oncol. 2010;46(11): 811-813.

20. Edwards PC, Kelsch R. Oral lichen planus: Clinical presentation and management. J Can Dent Assoc. 2002;68(8):494-499.

21. Ismail SB, Kumar SK, Zain RB. Oral lichen planus and lichenoid reactions: Etiopathogenesis, diagnosis, management and malignant transformation. J Oral Sci. 2007;49(2):89-106.

22. Rabinovich OF, Rabinovich IM, Guseva AV. Photodynamic therapy in treatment of severe oral lichen planus [in Russian]. Stomatologiia (Mosk). 2016;95(4):27-30.

23. Prażmo EJ, Kwaśny M, Łapiński M, Mielczarek A. Photodynamic therapy as a promising method used in the treatment of oral diseases. Adv Clin Exp Med. 2016;25(4):799-807

24. Vohra F, Al-Kheraif AA, Qadri T, et al. Efficacy of photodynamic therapy in the management of oral premalignant lesions. A systematic review. Photodiagnosis Photodyn Ther. 2015;12(1):150-159. doi: 10.1016/j.pdpdt.2014.10.001

25. Romeo U, Russo N, Palaia G, Tenore G, Del Vecchio A. Oral proliferative verrucous leukoplakia treated with the photodynamic therapy: A case report. Ann Stomatol (Roma). 2014;5(2):77-80.

26. Wong SJ, Campbell B, Massey B, et al. A phase I trial of aminolevulinic acid-photodynamic therapy for treatment of oral leukoplakia. Oral Oncol. 2013;49(9):970-976. doi: 10.1016/j.oraloncology.2013.05.011

27. Jerjes W, Upile T, Hamdoon Z, Mosse CA, Akram S, Hopper C. Photodynamic therapy outcome for oral dysplasia. Lasers Surg Med. 2011;43(3): 192-199. doi: 10.1002/Ism.21036

28. Maloth KN, Velpula N, Kodangal S, et al. Photodynamic therapy - a non-invasive treatment modality for precancerous lesions. J Lasers Med Sci. 2016;7(1):30-36. doi: 10.15171/jlms.2016.07

29. Pietruska M, Sobaniec S, Bernaczyk P, et al. Clinical evaluation of photodynamic therapy efficacy in the treatment of oral leukoplakia. Photodiagnosis Photodyn Ther. 2014;11(1):34-40. doi: 10.1016/j.pdpdt. 2013.10.003

30. Semkin VA, Rabinovich OF, Kasparov AS, Agapitova LP, Bezrukov AA. Analysis of comprehensive treatment of oral leukoplakia by laser ablation [in Russian]. Stomatologiia (Mosk). 2016;95(6):33-35.

31. Kawczyk-Krupka A, Waśkowska J, Raczkowska-Siostrzonek A, et al. Comparison of cryotherapy and photodynamic therapy in treatment of oral leukoplakia. Photodiagnosis Photodyn Ther. 2012;9(2):148-155. doi: 10.1016/j.pdpdt.2011.12.007 